\title{
Importância da avaliação funcional respiratória e motora em pacientes hemiparéticos por acidente vascular cerebral
}

\author{
Importance of respiratory and motor functional assessment \\ of hemiparetic stroke patients
}

\author{
Importancia de la evaluación funcional respiratoria y \\ motora en pacientes hemiparéticos debido a \\ accidente cerebrovascular
}

\author{
Letícia Ville Santos ${ }^{1}$, Fernando Luís Fischer Eichinger ${ }^{2}$, Fabrício \\ Noveletto ${ }^{3}$, Antonio Vinicius Soares ${ }^{4}$, Helton Eckermann da Silva ${ }^{5}$
}

1.Graduanda em Fisioterapia da Faculdade Guilherme Guimbala, Joinville-SC, Brasil.

2.Bacharel em Fisioterapia, Mestre em Ciências do Movimento Humano. Professor da Faculdade Guilherme Guimbala e da Universidade da Região de Joinville, Joinville-SC, Brasil.

3.Bacharel e Doutor em Engenharia Elétrica, Professor da Universidade do Estado de Santa Catarina CCT/UDESC, Joinville-SC, Brasil.

4.Bacharel em Fisioterapia, Doutor em Ciências do Movimento Humano). Professor da Universidade de Região de Joinville - UNIVILLE e da Faculdade IELUSC, Joinville-SC, Brasil.

5.Bacharel em Fisioterapia, Mestre em Saúde e Meio Ambiente. Professor da Faculdade Guilherme Guimbala, Joinville-SC, Brasil.

\section{Resumo}

Introdução. Hemiparéticos por Acidente Vascular Cerebral (AVC) apresentam diversas disfunções motoras e respiratórias, as quais estão relacionadas com prejuízos funcionais, levando a piora da qualidade de vida. Objetivo. Avaliar a função respiratória e motora de pacientes hemiparéticos por AVC e a correlação existente entre estas variáveis importantes para a capacidade funcional. Método. Trata-se de um estudo descritivo transversal, envolvendo 25 pacientes hemiparéticos pós-AVC. Foram avaliados: independência funcional (índice de Barthel modificado), força de preensão manual (dinamometria), velocidade de marcha, mobilidade funcional (Timed Up and Go Test), pico de fluxo expiratório (PFE - Peak Flow) e as pressões inspiratórias e expiratórias máximas (manovacuometria). Resultados. Foram constatados déficits nas variáveis motoras e respiratórias. Os valores de pressão respiratória foram inferiores quando comparados aos valores preditos para saudáveis, estando a pressão inspiratória máxima em $58,74 \%$ e a pressão expiratória máxima em $62,92 \%$ do valor predito $(p<0,001)$. Houve correlações entre PFE e todas as variáveis motoras apenas em pacientes agudos. Conclusão. Pacientes hemiparéticos apresentam importantes comprometimentos motores e respiratórios. A avaliação da musculatura respiratória pós-AVC, por vezes não realizada, é necessária na prática clínica pois fornece importantes informações sobre a função pulmonar, contribuindo para prevenção de complicações respiratórias nestes pacientes.

Unitermos. Testes de Função Respiratória; Desempenho Físico Funcional; Força Muscular; Hemiparesia; Acidente Vascular Cerebral

\footnotetext{
Abstract

Introduction. Hemiparetics post-stroke have several motor and respiratory dysfunctions, which are related to functional impairment, leading to a worse quality of life. Objective. To evaluate the respiratory and motor function of hemiparetic stroke patients and the correlation between these important variables for functional capacity. Method. This is a cross-sectional study involving 25 post-stroke hemiparetic patients. Functional independence (modified
} 
Barthel index), handgrip strength (dynamometry), gait speed, functional mobility (Timed Up and Go Test), peak expiratory flow (PEF) and maximal inspiratory and expiratory pressures (manovacuometry) were evaluated. Results. Deficits in motor and respiratory variables were found. The respiratory pressure values were lower when compared to the predicted values for healthy, with the maximum inspiratory pressure of $58.74 \%$ and the maximum expiratory pressure of $62.92 \%$ of the predicted value $(p<0.001)$. There were correlations between PEF and all motor variables only in acute patients. Conclusion. Hemiparetic patients have significant motor and respiratory impairment. The evaluation of respiratory muscle poststroke, sometimes not performed, is necessary in clinical practice because it provides important information on pulmonary function, contributing to the prevention of respiratory complications in these patients.

Keywords. Respiratory Function Tests; Physical Functional Performance; Muscle Strength; Hemiparesis; Stroke

\title{
Resumen
}

Introducción. Los hemiparéticos debido a un accidente cerebrovascular (ACV) tienen varias disfunciones motoras y respiratorias, que están relacionadas con el deterioro funcional, lo que lleva a una peor calidad de vida. Objetivo. evaluar la función respiratoria y motora de los pacientes con accidente cerebrovascular hemiparético y la correlación entre estas variables importantes para la capacidad funcional. Método. Este es un estudio transversal en el que participaron 25 pacientes hemiparéticos post accidente cerebrovascular. Se evaluaron la independencia funcional (índice de Barthel modificado), la fuerza de la empuñadura (dinamometría), la velocidad de la marcha, la movilidad funcional (prueba de tiempo y marcha), el flujo espiratorio máximo (PEF) y las presiones inspiratoria y espiratoria. valores máximos (manovacuometría). Resultados. Se encontraron déficits en las variables motoras y respiratorias. Los valores de presión respiratoria fueron más bajos en comparación con los valores predichos para salud, con la presión inspiratoria máxima en $58.74 \%$ y la presión espiratoria máxima en $62.92 \%$ del valor predicho $(p<0.001)$. Hubo correlaciones entre PEF y todas las variables motoras solo en pacientes agudos. Conclusión. los pacientes hemiparéticos tienen un deterioro motor y respiratorio significativo. La evaluación del músculo respiratorio después del accidente cerebrovascular, a veces no realizada, es necesaria en la práctica clínica porque proporciona información importante sobre la función pulmonar, contribuyendo a la prevención de complicaciones respiratorias en estos pacientes.

Palabras clave. Pruebas de función respiratoria; rendimiento físico funcional; fuerza muscular; hemiparesia; accidente cerebrovascular

Trabalho realizado na Associação Catarinense de Ensino, Faculdade Guilherme Guimbala - FGG. Curso de Fisioterapia. Núcleo de Pesquisa em Fisioterapia Cardiorrespiratória (NUPECRE). Joinville-SC, Brasil.

\section{INTRODUÇÃO}

\author{
O Acidente Vascular Cerebral (AVC) é um grave
} problema de saúde pública, considerado mundialmente como a segunda maior causa de morte e a primeira maior causa de invalidez, gerando um grande impacto econômico ${ }^{1}$. Entre as diversas manifestações clínicas presentes pósAVC, destaca-se que a disfunção motora é um dos problemas 
mais encontrados e consiste na consequência mais evidente após o AVC². A hemiparesia trata-se da perda parcial de força muscular (FM) no hemicorpo contralateral ao hemisfério afetado, sendo considerada como o quadro clínico típico da doença. Este quadro de fraqueza afeta mais de $80 \%$ dos pacientes, com consequente prejuízo da funcionalidade ${ }^{3}$.

A funcionalidade do sistema respiratório também é prejudicada em pacientes acometidos pelo AVC, visto que a respiração ocorre devido a ações de músculos estriados esqueléticos. Evidências apontam que aproximadamente $40 \%$ dos sobreviventes do AVC apresentam diminuição na mobilidade diafragmática, ocasionando perdas funcionais pulmonares de até $50 \%$ dos valores esperados pela idade 4 . Contudo, além da fraqueza muscular respiratória, é comum a existência de prejuízos na atividade dos músculos do tronco, redução da complacência torácica e pulmonar, podendo surgir mudanças no padrão respiratório, nos volumes e capacidades pulmonares ${ }^{5}$.

Esse conjunto de alterações prejudicam as funções protetoras do sistema respiratório, como a tosse, que para ser eficaz depende do fluxo de ar juntamente as altas pressões nas vias aéreas. Desta forma, a diminuição da pressão expiratória máxima (PEMáx) e da pressão inspiratória máxima (PIMáx), pode ser considerada um fator de risco para complicações respiratórias nestes pacientes ${ }^{4,6}$.

Como visto, as alterações motoras e respiratórias presentes em hemiparéticos pós-AVC, resultam em limitações funcionais evidenciadas por limitações ou até 
incapacidade na realização de atividades de vida diárias (AVDs). Estes prejuízos resultam em restrições na participação social e, consequentemente, na piora da qualidade de vida?.

Apesar de já existir uma ampla descrição na literatura sobre as disfunções motoras e consequentemente respiratórias pós-AVC, envolvendo a perda do controle voluntário e alteração do tônus muscular, a correlação entre alguns instrumentos de avaliação destas distintas variáveis respiratórias e motoras ainda é pouco conhecida. Existe uma escassez de estudos para alguns métodos avaliativos, sendo que o entendimento das associações entre determinados instrumentos é relevante para a compreensão das disfunções, assim como para a elaboração de um plano fisioterapêutico multidimensional.

Sendo assim, o objetivo deste estudo foi avaliar a função respiratória e motora de pacientes hemiparéticos acometidos por AVC e a correlação existente entre estas importantes variáveis para a capacidade funcional.

\section{MÉTODO}

\section{Amostra}

Este estudo foi aprovado pelo Comitê de Ética em Pesquisas Envolvendo Seres Humanos do Hospital Dona Helena e está registrado sob 0 número 12826219.6.0000.8062 (CAAE). 
Trata-se de um estudo descritivo correlacional com delineamento transversal, envolvendo 40 pacientes hemiparéticos por AVC, advindos do Ambulatório de Neurologia da Faculdade Guilherme Guimbala (FGG), localizado no município de Joinville (SC). Após os esclarecimentos quanto aos procedimentos todos os participantes assinaram o Termo de Consentimento Livre e Esclarecido (TCLE).

Foram adotados como critérios de inclusão: pacientes hemiparéticos por AVC, estáveis clinicamente; faixa etária a partir de 18 anos; ter manifestado apenas um evento; estar de acordo e mostrar interesse em participar do projeto do início ao término, assinando o TCLE. Já como critérios de exclusão foram estabelecidos: hemiparesia decorrente de outras doenças que não sejam o AVC, bem como hemiplegia; comprometimento motor bilateral, que impeça a caracterização como hemiparético; comprometimento visual e/ou auditivo grave; pacientes não cooperativos e/ou com déficit cognitivo grave avaliado através do Mini Exame do Estado Mental ${ }^{8}$; incapacidade de adotar a bipedestação de maneira independente (podendo ser utilizados dispositivos de auxílio: muleta, bengala ou andador); que estejam realizando algum tipo de reabilitação para função respiratória; traqueostomizados ou que utilizem suporte de oxigênio; incapacidade de tolerem o uso de clipe nasal durante a realização dos testes.

Um questionário foi previamente aplicado para coletar todas as informações relevantes para realização do projeto. 
Após a aplicação do questionário, procederam-se as demais avaliações. Cada avaliação tinha duração média de uma hora. Foram utilizados os seguintes testes e instrumentos:

- Índice de Barthel Modificado (IBM): para avaliação da independência funcional. Consiste em dez itens contendo AVDs e mobilidade, onde a pontuação é baseada no nível de assistência necessária para completar a tarefa totalizando até 100 pontos, onde 0 indica dependência total e 100 a independência total ${ }^{9}$;

- Mini Exame do Estado Mental (MEEM): para avaliar o nível cognitivo dos pacientes. Destaca-se que este instrumento foi utilizado apenas para triagem dos pacientes, sendo adotados os pontos de corte propostos por Bertolucci et al. (1994), que estão relacionados ao grau de escolaridade $^{8}$;

- Dinamometria (DIN): para mensuração da força de preensão manual (FPM). Possui fácil aplicação, sendo considerada o padrão ouro para mensuração de força muscular de toda a extremidade superior em pessoas saudáveis ou com doenças neurológicas ${ }^{7,10}$. Foi utilizado o dinamômetro digital de preensão manual portátil da ECLEAR ${ }^{\circledR}$, modelo $\mathrm{EH} 101$, com capacidade máxima de $90 \mathrm{~kg}$ e escala de 1 grama, devidamente calibrado. Para a realização do teste, o paciente era posicionado conforme recomendação da American Society of Hand Therapists (ASHT): sentado com os quadris e joelhos a $90^{\circ}$ de flexão, ombro em adução, cotovelo fletido a $90^{\circ}$, antebraço e punho em posição neutra (medioprono), enquanto o examinador 
sustentava o equipamento. Foram realizadas três medidas com descanso de aproximadamente 30 segundos entre elas, sendo o paciente orientado a realizar sempre o máximo de força possível (utilizada a média destas medidas com referência para o estudo). Cabe destacar que o equipamento teve a empunhadura ajustada de acordo com as características antropométricas de cada paciente;

- Teste de Velocidade da Marcha: para avaliar a velocidade de marcha (VM), que é a mais objetiva e simples medida de desempenho locomotor ${ }^{11}$. Foi solicitada a realização do teste o mais rápido possível, sendo adotado um percurso de 10 metros para cronometragem. Foram acrescidos 3 metros no início e no final do percurso, para eliminar os efeitos da aceleração e desaceleração no teste. Os pacientes foram orientados a caminhar o mais rápido possível, podendo ser utilizado dispositivo para o auxílio da marcha. O cálculo para obtenção da VM é o tempo gasto para realização da tarefa dividido pela distância percorrida durante a tarefa (10 metros).

- $\quad$ Timed Up and Go Test (TUGT) para avaliação da mobilidade funcional, por meio da mensuração do tempo de realização da tarefa proposta (paciente se levantar de uma cadeira, caminhar em linha reta por 3 metros, virar, caminhar de volta para a cadeira e sentar-se) ${ }^{12}$;

- Peak Flow (PF): para medir a capacidade de gerar fluxo expiratório adequado para proteção das vias aéreas. Durante a medida do pico de fluxo expiratório (PFE), a expiração, mesmo sendo um processo passivo, em pacientes 
com doenças cerebrovasculares é realizada de forma deficiente devido à diminuição da atividade e alteração do tônus dos músculos abdominais, que são essenciais na expiração forçada ${ }^{13}$. Foi utilizado um medidor de PFE da marca Asses ${ }^{\circledR}$. Com o participante sentado utilizando o clipe nasal, foi solicitado que realizasse uma expiração forçada e rápida, que conseguisse ser mantida de um a dois segundos, respeitando o intervalo de um minuto entre uma aferição e outra. Para tais mensurações foram utilizados bocais descartáveis mantendo a higiene perante os procedimentos. A medida foi repetida até que três leituras não tivessem diferença maior que $10 \%$ uma da outra.

- Manovacuometria (MV): para avaliação da força dos músculos respiratórios. Foram utilizadas medidas estáticas das pressões respiratórias máximas por meio de um manovacuômetro aneróide da marca Suporte - Indústria Brasileira ${ }^{\circledR}$, graduado em centímetros de água $\left(\mathrm{cmH}_{2} \mathrm{O}\right)$, com orifício de $2 \mathrm{~mm}$ para evitar a interferência do uso de músculos bucinadores durante medidas de pressões expiratórias. A aferição da PIMáx foi realizada solicitando ao participante que expirasse todo o ar de forma completa e passiva, até que chegasse no volume residual e imediatamente inspirasse o mais rápido e forte possível, ocluindo a entrada de ar gerando uma manobra de Muller. Na mensuração da PEMáx o participante realizava uma inspiração completa sem forçar, seguida da maior expiração forçada possível (manobra de Valsalva). Em ambas medidas os pacientes foram encorajados durante a manobra. Foram 
realizadas no máximo 5 repetições até que se obtivessem valores com diferença menor que $10 \%$ entre elas, não sendo o último resultado maior que o primeiro, sempre respeitando um intervalo de um minuto entre cada medição. Em posse das três medidas, a maior foi utilizada. O clipe nasal foi utilizado nas aferições conforme recomendações da Sociedade Brasileira de Pneumologia e Tisiologia14,15.

\section{Análise Estatística}

Os dados foram analisados por meio do software Graph Pad Prism $6^{\circledR}$. Foi utilizada estatística descritiva (média, desvio padrão, valores máximos e mínimos) e distribuição de frequências com valores absolutos e percentuais, Teste de Shapiro-Wilk (verificação da normalidade dos dados), teste $t$ de Student Independente (para comparação dos valores obtidos nos testes dos pacientes com os valores preditos para saudáveis) o teste de correlação de Pearson (para analisar a relação entre as medidas motoras e respiratórias). Foi adotado um nível de significância de 5\%.

\section{RESULTADOS}

Foram avaliados 40 pacientes, porém, 15 foram excluídos por não se enquadrarem nos critérios adotados no estudo. Assim, a população elegível foi composta por 25 pacientes hemiparéticos por AVC (10 do sexo masculino), com idade média de $61,1 \pm 13,1$ anos e com tempo médio

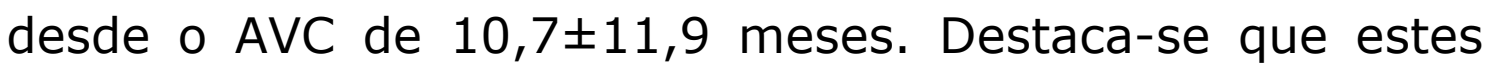


pacientes foram divididos em dois grupos para análise: Grupo de Pacientes Agudos (GA) e Grupo de Pacientes Crônicos (GC). Em ambos os grupos foi constatado um predomínio do AVC tipo isquêmico (Agudos=85,0\% e Crônicos $=42,0 \%$ ), além de existir uma prevalência do quadro de hemiparesia no dimídio corporal esquerdo. A Tabela 1 apresenta a caracterização geral dos participantes do GA e do GC.

Tabela 1. Caracterização geral dos participantes.

\begin{tabular}{|c|c|c|c|c|}
\hline \multirow{2}{*}{ Características $(n=25)$} & \multicolumn{2}{|c|}{ GA $(n=13)$} & \multicolumn{2}{|c|}{ GC $(n=12)$} \\
\hline & $\overline{\mathbf{x}} \pm \mathbf{s}$ & f (\%) & $\overline{\mathbf{x}} \pm \mathbf{s}$ & f (\%) \\
\hline \multicolumn{5}{|l|}{ Sexo } \\
\hline Feminino & --- & $8(62,0)$ & --- & $7(58,0)$ \\
\hline Masculino & --- & $5(38,0)$ & --- & $5(42,0)$ \\
\hline $\begin{array}{l}\text { Idade (anos completos) } \\
\text { Etnia autorreferida }\end{array}$ & \multicolumn{3}{|c|}{ Etnia autorreferida } & --- \\
\hline Branco & --- & $12(92,0)$ & --- & $10(83,0)$ \\
\hline Negro & --- & $1(8,0)$ & --- & $2(17,0)$ \\
\hline $\begin{array}{l}\text { IMC }\left(\mathrm{kg} / \mathrm{m}^{2}\right) \\
\text { Lateralidade autorrelatada }\end{array}$ & $28,7 \pm 5,8$ & --- & $27,6 \pm 4,8$ & ---- \\
\hline Destro & --- & $11(85,0)$ & ---- & $8(67,0)$ \\
\hline Sinistro/Canhoto & --- & $2(15,0)$ & --- & $4(33,0)$ \\
\hline \multicolumn{5}{|l|}{ Hemiparesia } \\
\hline Esquerda & --- & $9(69,0)$ & ---- & $7(58,0)$ \\
\hline Direita & --- & $4(31,0)$ & --- & $5(42,0)$ \\
\hline $\begin{array}{l}\text { Tempo de AVC (meses) } \\
\text { Tipo de AVC }\end{array}$ & $1,8 \pm 0,8$ & & $20,3 \pm 10,9$ & \\
\hline Isquêmico & --- & $11(85,0)$ & ---- & $7(58,0)$ \\
\hline Hemorrágico & --- & $2(15,0)$ & --- & $5(42,0)$ \\
\hline
\end{tabular}

As medidas de comparação das variáveis relacionadas à caraterização geral (Tabela 1) e ao perfil clínico dos participantes (Tabela 2), entre o GA e o GC, revelaram que não existiam diferenças significantes entre os grupos em nenhuma das variáveis. 
Tabela 2. Caracterização dos participantes quanto ao perfil clínico.

\begin{tabular}{lccc}
\hline Características $(\mathbf{n = 2 5})$ & $\begin{array}{c}\text { GA }(\mathbf{n = 1 3}) \\
\overline{\mathbf{x}} \pm \mathbf{s}\end{array}$ & $\begin{array}{c}\text { GC }(\mathbf{n}=\mathbf{1 2}) \\
\overline{\mathbf{x}} \pm \mathbf{s}\end{array}$ & p-valor \\
\hline IBM (pontos) & $82,3 \pm 13,2$ & $82,5 \pm 14,2$ & 0,972 \\
DIN-P (kgf) & $19,4 \pm 12,8$ & $13,5 \pm 8,4$ & 0,192 \\
TUGT (s) & $17,7 \pm 8,8$ & $26,7 \pm 20,8$ & 0,166 \\
VM (m/s) & $0,86 \pm 0,47$ & $0,80 \pm 0,58$ & 0,765 \\
PFE (L/min) & $277,4 \pm 100,7$ & $292,5 \pm 94,0$ & 0,703 \\
PIMáx (cmH $\left.\mathrm{cm}_{2} \mathrm{O}\right)$ & $56,1 \pm 27,3$ & $46,9 \pm 24,3$ & 0,382 \\
PEMáx $\left(\mathrm{cmH}_{2} \mathrm{O}\right)$ & $60,1 \pm 25,0$ & $54,0 \pm 26,3$ & 0,558 \\
\hline
\end{tabular}

Nível de significância $\mathrm{p}<0,05 ; \mathrm{p}$ : probabilidade de significância obtida pelo teste $\mathrm{t}$ independente; GA: Grupo de Pacientes Agudos; GC: Grupo de Pacientes Crônicos; IBM: índice de barthel modificado; DIN-P: dinamometria no membro parético; TUGT: timed up and go test; VM: velocidade de marcha; PF: peak flow; PIMáx: pressão inspiratória máxima; PEMáx: pressão expiratória máxima; n: amostra total.

A avaliação da função respiratória foi realizada por meio do PF medindo o PFE e a MV verificando a PIMáx e PEMáx. Os resultados obtidos foram comparados com os valores preditos para população saudável de acordo com os estudos de Teixeira Salmela et al. (2005) ${ }^{16}$ e Neder et al. (1999) ${ }^{17}$. A Tabela 3 apresenta os resultados dos testes respiratórios e as comparações com os valores preditos para saudáveis, em agudos e crônicos.

Tabela 3. Comparação das medidas respiratórias de PFE, PIMáx e PEMáx com os valores preditos para saudáveis, em agudos e crônicos.

\begin{tabular}{lcccc}
\hline Variável & $\begin{array}{c}\text { Valor obtido } \\
(\overline{\mathbf{x}} \pm \mathbf{s})\end{array}$ & $\begin{array}{c}\text { Valor predito } \\
(\overline{\mathbf{x}} \pm \mathbf{s})\end{array}$ & $\begin{array}{c}\text { Percentual } \\
\text { do predito }\end{array}$ & p-valor \\
\hline GA (n=13) & & & & \\
PFE (L/min) & $277,4 \pm 100,7$ & $421,4 \pm 80,5$ & 65,83 & $<0,001$ \\
PIMáx (cmH $\mathbf{0})$ & $-56,1 \pm 27,3$ & $-88,0 \pm 19,1$ & 63,75 & $<0,001$ \\
PEMáx (cmH20) & $60,1 \pm 25,0$ & $96,0 \pm 38,3$ & 62,60 & $<0,001$ \\
GC (n=12) & & & & \\
PFE (L/min) & $292,5 \pm 94,0$ & $442,7 \pm 67,1$ & 66,07 & $<0,001$ \\
PIMáx (cmH20) & $-46,9 \pm 24,3$ & $-93,1 \pm 17,7$ & 50,38 & $<0,001$ \\
PEMáx (cmH20) & $54,0 \pm 26,3$ & $95,8 \pm 23,0$ & 56,37 & $<0,001$ \\
\hline
\end{tabular}

Nível de significância $p<0,05 ; p$ : probabilidade de significância obtida pelo teste $t$ independente; GA: Grupo de Pacientes Agudos; GC: Grupo de Pacientes Crônicos; PFE: pico de fluxo expiratório; PIMáx: pressão inspiratória máxima; PEMáx: pressão expiratória máxima; n: amostra total. 
Em ambos os grupos pode ser observada uma diferença significante entre os valores obtidos e os preditos para a população saudável, sendo os valores obtidos muito inferiores (GA: 65,83\% no PFE, 63,75\% na PIMáx e 62,60\% na PEMáx / GC: 66,07\% no PFE, 50,38\% na PIMáx e 56,37\% na PEMáx). Desta forma, foi constatado que os pacientes em questão, realmente apresentam déficits importantes nas medidas respiratórias, quando comparados à saudáveis de faixa etária semelhante.

Não foram observadas correlações significantes entre as variáveis de função respiratória e as medidas de desempenho motor ao se analisar toda a amostra. Porém, quando realizada estratificação de acordo com a fase da doença (agudos e crônicos), constataram-se correlações fortes e moderadas entre o PF e as variáveis VM, TUGT, DIN e IBM, apenas nos pacientes agudos. Os resultados das análises de correlação realizadas com os pacientes agudos estão apresentados na Tabela 4.

Tabela 4. Correlação entre as medidas de função respiratória e de desempenho motor em hemiparéticos na fase aguda.

\begin{tabular}{lcccc}
\hline Variável $(\mathbf{n = 1 3})$ & VM $(\mathbf{m} / \mathbf{s})$ & TUGT $(\mathbf{s})$ & DIN-P (kgf) & IBM \\
\hline PFE $(\mathbf{L} / \mathbf{m i n})$ & $0,72^{*}$ & $-0,63^{*}$ & $0,67 *$ & $0,71^{*}$ \\
PIMáx $\left(\mathbf{c m H}_{2} \mathbf{O}\right)$ & $-0,01$ & $-0,06$ & 0,28 & 0,04 \\
PEMáx $\left(\mathbf{c m H}_{\mathbf{2}} \mathbf{0}\right)$ & 0,50 & $-0,30$ & 0,28 & 0,20 \\
\hline
\end{tabular}

Nível de significância $p<0,05$ (Correlação de Pearson); * correlação significativa; PFE: pico de fluxo expiratório; PIMáx: pressão inspiratória máxima; PEMáx: pressão expiratória máxima; VM: velocidade de marcha; TUGT: timed up and go test; DIN-P: dinamometria no membro parético; IBM: índice de barthel modificado; amostra total $(n)$. 


\section{DISCUSSÃO}

A hemiparesia pós-AVC caracteriza-se pela fraqueza muscular do hemicorpo contralateral à lesão encefálica, incluindo os músculos responsáveis pela respiração ${ }^{13}$. Esta redução da FM gera alterações biomecânicas, que comprometem a atividade dos músculos inspiratórios e expiratórios do tórax e do abdômen, dificultando a função pulmonar7. Assim, este conjunto de mudanças presentes nestes pacientes repercute em prejuízos funcionais motores e respiratórios, os quais pioram a qualidade de vida do indivíduo ${ }^{4}$.

No presente estudo, as avaliações realizadas revelaram que os pacientes apresentavam comprometimentos importantes tanto nas variáveis motoras (DIN, VM e TUGT) quanto nas variáveis respiratórias (PFE, PIMáx e PEMáx), na fase aguda e na fase crônica da doença. Desta forma, é imprescindível a realização de uma avaliação funcional respiratória, além da avaliação motora comumente realizada.

Os diversos comprometimentos observados em pacientes pós-AVC, resultam em um impacto da funcionalidade. Nesse sentido, o IBM é um importante instrumento de avaliação destes pacientes, pois permite mensurar o nível de independência do sujeito e o grau de assistência exigido na realização de diferentes AVDs $^{9}$. A pontuação obtida neste instrumento permite classificar os pacientes com: total dependência (abaixo de 20), moderada dependência (20-55), leve dependência (60-95) ou total 
independência $(100)^{18}$. Os pacientes do presente estudo apresentaram pontuações no IBM, que indicam leve

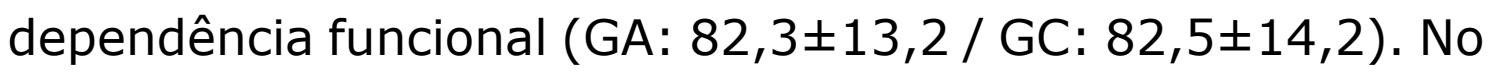
entanto as demais avaliações motoras realizadas, mostraram importantes déficits nestes pacientes.

Como já descrito anteriormente, a fraqueza muscular está presente na maioria dos pacientes que sofreram AVC, estando relacionada com perdas funcionais, evidenciadas na realização das $\mathrm{AVDs}^{19}$. Neste sentido, a FPM tem sido sugerida como uma variável importante a ser avaliada pósAVC, pois permite mensurar o nível de FM do sujeito. Um estudo buscando estabelecer valores normativos de FPM para sujeitos saudáveis de diferentes faixas etárias observou medidas de 2629 pessoas (1314 homens), constatando-se em indivíduos com faixa etária entre 50 e 70 anos valores de

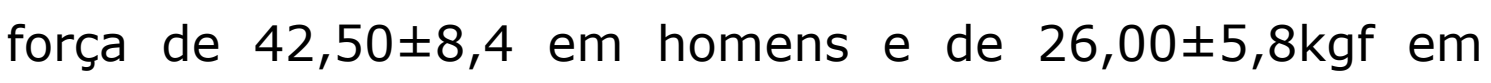
mulheres ${ }^{20}$. Comparando os valores de DIN dos pacientes envolvidos em nossa pesquisa $(19,4 \pm 12,8$ e $13,5 \pm 8,4$, em GA e GC, respectivamente) com os valores normativos apresentados para pessoas saudáveis, percebe-se que os hemiparéticos por AVC possuem níveis inferiores nesta variável, corroborando com outros estudos da literatura $7,10,19$.

A VM é uma medida muito utilizada para a avaliação do equilíbrio e da mobilidade física em pacientes vítimas de $A V C^{21}$. Trata-se de um instrumento bastante confiável, válido, sensível e específico, que está relacionado com a capacidade funcional, e por estes motivos, considerado como 
o "sexto sinal vital"11. Indivíduos que apresentam VM entre 0 e $0,6 \mathrm{~m} / \mathrm{s}$ podem ser caracterizados como dependentes nas AVDs e nas atividades instrumentais da vida diária, além de apresentarem maior probabilidade de internações. Em contrapartida, os que apresentam VM superior a $1,0 \mathrm{~m} / \mathrm{s}$ podem ser considerados independentes nas AVDs e apresentam menor probabilidade de internações ${ }^{11}$. Na população brasileira com faixa etária entre 40 e 70 anos, foram descritos como valores preditos para VM 1,34m/s para homens e $1,27 \mathrm{~m} / \mathrm{s}$ para mulheres ${ }^{21}$. Os pacientes do

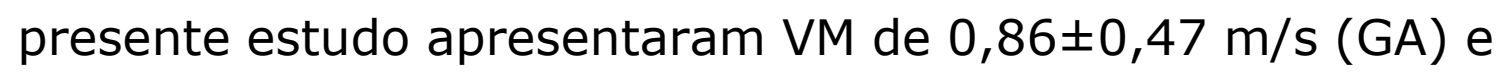
$0,80 \pm 0,58 \mathrm{~m} / \mathrm{s}$ (GC), o que indica a presença de limitações locomotoras, que indicam um maior risco de quedas.

O TUGT é um teste muito conhecido e utilizado mundialmente, por se tratar de uma medida altamente confiável e fornecer uma importante informação sobre a mobilidade funcional. Os valores referenciais deste instrumento para a população saudável na faixa etária de 60 a 79 anos encontram-se entre 8,1 e 9,2 segundos ${ }^{22}$. Um resultado do TUGT entre 10 e 20 segundos sugere uma independência para transferências básicas, com uma ou duas atividades de vida diária comprometidas ${ }^{12}$. Além disso, tempos superiores a 14 segundos são indicativos de um risco de quedas em pacientes com AVC 23 . Tendo em vista os dados mencionados, nota-se que os participantes avaliados no presente estudo possuem déficits da mobilidade funcional, com limitações em AVDs e maior risco de quedas, pois 
tiveram desempenho médio no TUGT de 17,7 segundos (GA) e 26,7 segundos (GC).

Como já descrito na literatura, o quadro de hemiparesia pós-AVC limita a mobilidade no hemicorpo contralateral à lesão, o que resulta em uma hipofunção diafragmática na cúpula do lado parético. Assim, existe um desequilíbrio muscular causado pela redução da ativação dos músculos abdominais, o qual altera o posicionamento da caixa torácica, sendo responsável pela força muscular respiratória reduzida nestes pacientes, quando comparada aos valores referenciais para indivíduos saudáveis ${ }^{13}$.

A deficiência respiratória pós-AVC foi investigada em alguns estudos que compararam as pressões máximas respiratórias dos pacientes com os valores preditivos, sendo encontrada diminuição destas variáveis nos indivíduos avaliados, corroborando com os resultados do presente estudo $7,16,24$. Foi encontrada uma fraqueza muscular inspiratória (PIMáx) em $66,7 \%$ de uma amostra de indivíduos com AVC, assim como uma FM expiratória (PEMáx) e PFE abaixo dos valores referenciais em $16,7 \%$ dos indivíduos avaliados ${ }^{25}$.

O comprometimento da FM respiratória por inadequado disparo neural e uma coordenação muscular inefetiva, compromete também a função de proteção e remoção de secreção das vias aéreas. A alta pressão intrapulmonar após uma inspiração profunda, fechamento da glote e contração da musculatura expiratória proporcionam um pico de fluxo de tosse (PFT), diretamente ligado aos valores de PEMáx e 
PFE. Sendo que, uma PEMáx entre $45 \mathrm{cmH}_{2} \mathrm{O}$ e $60 \mathrm{cmH}_{2} \mathrm{O}$ e um valor abaixo de $160 \mathrm{~L} / \mathrm{min}$ para o PFE estão relacionados a uma baixa eficiência de tosse ${ }^{26}$. Foi encontrada uma redução dos valores do PFT em 19 pacientes pós-AVC, demonstrando o comprometimento da função da tosse. Assim como os resultados do presente estudo, foi observada uma PEMáx diminuída, incapaz de gerar um PFE adequado ${ }^{27}$.

No que diz respeito a correlação entre as variáveis respiratórias (PFE, PIMáx e PEMáx) e os testes de desempenho funcional (IBM, DIN, VM e TUGT) em vítimas de AVC, a literatura aponta a existência de correlação apenas com a FPM avaliada com a DIN. Foi encontrada uma correlação entre a FPM com PFE e PEMáx ( $r=0,5 \mathrm{p}=0,000$ e $r=0,3 p=0,012$, respectivamente) em hemiparéticos por AVC, em fase crônica da doença, o que contrasta o presente estudo, no sentido de encontrarmos correlação apenas com PFE em indivíduos com AVC em fase aguda28. Quanto as outras variáveis, segundo a literatura, apenas o IBM e o TUGT foram correlacionados com as medidas respiratórias, porém em idosos hígidos ou em indivíduos com doença pulmonar obstrutiva crônica ${ }^{29}$. A maioria dos estudos encontrados envolvendo hemiparéticos por AVC em diferentes fases de convalescência, apontam correlação entre testes de controle de tronco e variáveis respiratórias como a PIMáx ${ }^{30}$, PEMáx e PFE ${ }^{13}$.

Como pode-se constatar, há divergência entre as publicações que citam correlação entre variáveis motoras e respiratórias, em relação ao tempo de injúria encefálica pós- 
AVC. Nos pacientes deste estudo, foram encontradas correlações significativas (fortes e moderadas) entre o PFE e as medidas de desempenho motor (IBM, DIN, VM e TUGT) apenas nos indivíduos com AVC em fase aguda.

É conhecido que a maioria das melhorias na função motora geral ocorre durante o primeiro ano após o AVC, com menor progresso após 6 meses e uma função motora razoavelmente estável a partir dos 12 meses após o AVC ${ }^{19}$. Nesse sentido, considera-se que a correlação entre função motora e força respiratória em fase crônica da doença, pode não ser significativa, devido às estratégias compensatórias para manter a função respiratória, como um maior recrutamento dos músculos do lado não parético e uso da musculatura acessória, apesar da instabilidade proximal ${ }^{31}$. Porém, os dados do presente estudo devem ser analisados com cautela, tendo em vista o tamanho da amostra.

\section{CONCLUSÃO}

Os pacientes hemiparéticos por AVC em fase aguda ou crônica, estudados na presente pesquisa, apresentaram além de déficits da função motora, comprometimentos importantes da função respiratória. Desta forma, salienta-se que a avaliação da função respiratória, por vezes não realizada, se faz necessária na prática clínica, pois fornece importantes informações sobre a função pulmonar, contribuindo para prevenção de complicações respiratórias nestes pacientes. 
Foram observadas correlações significantes entre a variável respiratória do PFE e as variáveis motoras (IBM, DIN, TUGT e VM) apenas nos indivíduos em fase aguda, após o AVC. Tal achado indica que deve existir uma atenção ainda maior nos componentes da função respiratória nesta fase inicial após a ocorrência do evento.

Os achados do estudo reforçam a importância da avaliação da função respiratória em indivíduos com AVC, como rotina clínica, para melhor compreensão das disfunções e elaboração de um plano fisioterapêutico multidimensional, não apenas voltado para as disfunções motoras.

\section{REFERÊNCIAS}

1.Ildstad F, Ellekjar $\mathrm{H}$, Wethal T, Lydersen S, Kutschera JS, Fjaertoft $\mathrm{H}$, et al. Stroke risk after transient ischemic attack in a Norwegian prospective cohort. BMC Neurol 2019;19:2. http://dx.doi.org/10.1186/s12883-018-1225-y

2.Dorsch S, Ada L, Canning CG. Lower limb strength is significantly impaired in all muscle groups in ambulatory people with chronic stroke: a cross-sectional study. Arch Phys Med Rehabil 2016;97:522-7. http://dx.doi.org/10.1016/j.apmr.2015.10.106

3. Najafi Z, Rezaeitalab F, Yaghubi M, Manzari ZS. The effect of biofeedback on the motor - muscular situation in rehabilitation of stroke patients: a randomized controlled trial. J Caring Sci 2018; 7:8993. http://dx.doi.org/10.15171/jcs.2018.014

4.Lee $K$, Park D, Lee G. Progressive respiratory muscle training for improving trunk stability in chronic stroke survivors: a pilot randomized controlled trial. J Stroke Cerebrovasc Dis 2019;28:1200-11. http://dx.doi.org/10.1016/j.jstrokecerebrovasdis.2019.01.008

5. Lee DK, Kim SH. The effect of respiratory exercise on trunk control, pulmonary function, and trunk muscle activity in chronic stroke patients. J Phys Ther Sci 2018;30:700-3. http://dx.doi.org/10.1589/jpts.30.700

6. Caruso P, Albuquerque AL, Santana PV, Cardenas LZ, Ferreira JG, Prina $\mathrm{E}$, et al. Diagnostic methods to assess inspiratory and expiratory 
muscle strength. J Bras Pneumol 2015;41:110-23. http://dx.doi.org/10.1590/S1806-37132015000004474

7.Silva SM, Corrêa JCF, Silva C, Silva PFC, Corrêa FI. Relação entre a força de preensão manual e capacidade funcional após acidente vascular cerebral. Rev Neurocienc 2014;23:74-80. https://doi.org/10.34024/rnc.2015.v23.8052

8.Bertolucci PHF, Brucki SMD, Campacci SR, Juliano YO. Mini-exame do estado mental em uma população geral: impacto da escolaridade. Arq Neuropsiquiatr 1994;52:1-7. https://doi.org/10.1590/S0004282X1994000100001

9.Cincura C, Pontes Neto OM, Neville IS, Mendes HF, Menezes DF, Mariano DC, et al. Validation of the national institutes of health stroke scale, modified rankin scale and barthel index in Brazil: the role of cultural adaptation and structured interviewing. Cerebrovasc Dis 2009;27:119-22. http://dx.doi.org/10.1159/000177918

10.Ekstrand E, Lexell J, Brogårdh C. Grip strength is a representative measure of muscle weakness in the upper extremity after stroke. Top Stroke Rehabil $2016 ; 23: 400-5$ http://dx.doi.org/10.1080/10749357.2016.1168591

11.Fritz S, Lusardi M. White paper: "walking speed: the sixth vital sign". J Geriatr Phys Ther 2009;32:46-9. https://journals.Iww.com/jgpt/fulltext/2009/32020/white_paper_w alking speed the sixth vital sign .2.aspx

12.Podsiadlo D, Richardson S. The timed up and go: a test of basic functional, ability for frail elderly persons. J Am Geriatr Soc 1991;39:142-8.

5415.1991.tb01616.x

13.Jandt S, Caballero R, Forgiarini J, Alberto L, Dias A. Correlation between trunk control, respiratory muscle strength and spirometry in patients with stroke: An observational study. Physiother Res Int 2011;16:218-24. http://dx.doi.org/10.1002/pri.495

14.Pessoa IMBS, Houri Neto M, Montemezzo LA, Silva LAM, Andrade $A D$, Parreira VF. Predictive equations for respiratory muscle strength according to international and Brazilian guidelines. Braz J Phys Ther 2014;18:410-8. http://dx.doi.org/10.1590/bjpt-rbf.2014.0044

15.Leiner GC, Abramowitz S, Small MJ, Stenby VB, Lewis WA. Expiratory peak flow rate. Standard values for normal subjects. Use as a clinical test of ventilatory function. Am Rev Respir Dis 1963;88:64451. http://dx.doi.org/10.1164/arrd.1963.88.5.644

16. Teixeira Salmela LF, Parreira VF, Britto RR, Brant TC, Inácio EP, Alcântara TO, et al. Respiratory pressures and thoracoabdominal motion in community-dwelling chronic stroke survivors. Arch Phys Med Rehabil

http://dx.doi.org/10.1016/j.apmr.2005.03.035

2005;86:1974-8.

17. Neder JA, Andreoni S, Lerario MC, Nery LE. Reference values for lung function tests: II. Maximal respiratory pressures and voluntary ventilation. Braz J Med Biol Res 1999;32:719-27. http://dx.doi.org/10.1590/S0100-879X1999000600007 
18. Torres GV, Reis LA. Assessment of functional capacity in elderly residents of an outlying area in the hinterland of Bahia/Northeast Brazil. Arq Neuropsiquiatr 2010;68:39-43. http://dx.doi.org/10.1590/S0004-282X2010000100009

19.Stock R, Thrane G, Askim T, Anke A, Mork PJ. Development of grip strength during the first year after stroke. J Rehabil Med 2019;51:24856. http://dx.doi.org/10.2340/16501977-2530

20.Massy Westropp NM, Gill TK, Taylor AW, Bohannon RW, Hill CL. Hand grip strength: age and gender stratified normative data in a population-based study. BMC Res Notes 2011;4:127-31. http://dx.doi.org/10.1186/1756-0500-4-127

21. Novaes RD, Miranda AS, Dourado VZ. Velocidade usual da marcha em brasileiros de meia idade e idosos. Rev Bras Fisioter 2011;15:11722. http://dx.doi.org/10.1590/S1413-35552011000200006

22.Bohannon RW. Reference values for the timed up and go test: a descriptive meta-analysis. J Geriatr Phys Ther 2006;29:64-8. http://dx.doi.org/10.1519/00139143-200608000-00004

23.Andersson $\AA$ G, Kamwendo K, Seiger $\AA$, Appelros P. How to identify potential fallers in a stroke unit: validity indexes of four test methods. J Rehabil Med 2006;38:186-91.

http://dx.doi.org/10.1080/16501970500478023

24. Meneghetti C, Figueiredo V, Guedes C, Batistela A. Avaliação da força muscular respiratória em indivíduos acometidos por Acidente Vascular Cerebral. Rev Neurocienc 2011;19:56-60. https://doi.org/10.34024/rnc.2011.v19.8398

25.Zaleski TDP, Camera F, Wisniewski E, Wisniewski M. Avaliação da força muscular respiratória e função pulmonar em indivíduos com acidente vascular cerebral. Perspectiva 2018;42:15-22. http://www.uricer.edu.br/site/pdfs/perspectiva/1005 655.pdf

26. Nagato AC, Nunes LAS, Dourado VA, Diniz MF, Silva MAS, Dornelas $\mathrm{G}$, et al. Correlação entre a pressão expiratória máxima (PEmáx) e pico de fluxo expiratório máximo (PFE) em indivíduos saudáveis. Rev Int Est Exp 2012;4:7-15. https://periodicos.ufjf.br/index.php/riee/article/view/23989

27.Sartor MM, Guillen Solà A, Ramirez Fuentes C, Duarte E, Marco E. Peak expiratory cough flow and respiratory muscle function in acute stroke patients. Eur Respir J 2017;50: PA3696. http://dx.doi.org/10.1183/1393003.congress-2017.PA3696

28. Kim NS. Correlation between grip strength and pulmonary function and respiratory muscle strength in stroke patients over 50 years of age. J Exerc Rehabil 2018;14:1017-23. http://dx.doi.org/10.12965/jer.1836444.222

29. Reis L, Moreira J, Duarte S. Risk of falling, respiratory and functional capacity in elderly residents in institutions of long stay. Rev Neurocienc 2015;23(3):397-404. https://doi.org/10.34024/rnc.2015.v23.8008 30.Santos RS, Dall'alba SCF, Forgiarini SGI, Rossato D, Dias AS, Forgiarini Júnior $A$. Relationship between pulmonary function, functional independence, and trunk control in patients with stroke. Arq 
Neuropsiquiatr 2019;77:387-92. http://dx.doi.org/10.1590/0004$\underline{282 \times 20190048}$

31.Pompeu S, Pompeu J, Rosa M, Silva M. Correlação entre função motora, equilíbrio e força respiratórios pós acidente vascular cerebral. Rev Neurocienc 2011;19:614-20.

https://doi.org/10.34024/rnc.2011.v19.8324 